\title{
Pure Collective Precession Motion of High-Spin Torus Isomer
}

\author{
T. Ichikawa, ${ }^{1}$ K. Matsuyanagi, ${ }^{1,2}$ J. A. Maruhn, ${ }^{3}$ and N. Itagaki ${ }^{1}$ \\ ${ }^{1}$ Yukawa Institute for Theoretical Physics, Kyoto University, Kyoto 606-8502, Japan \\ ${ }^{2}$ RIKEN Nishina Center, Wako 351-0198, Japan \\ ${ }^{3}$ Institut fuer Theoretische Physik, Universitaet Frankfurt, D-60438 Frankfurt, Germany
}

(Dated: December 26, 2017)

\begin{abstract}
We investigate the precession motion of the exotic torus configuration in high-spin excited states of ${ }^{40} \mathrm{Ca}$. For this aim, we use the three-dimensional time-dependent Hartree-Fock (TDHF) method. Although the high-spin torus isomer is a unique quantum object characterized by the alignment of angular momenta of independent single-particle motions, we find that the obtained moment of inertia for rotations about an axis perpendicular to the symmetry axis is close to the rigid-body value. We also analyze the microscopic structure of the precession motion using the random-phase approximation (RPA) method for high-spin states. In the RPA calculation, the precession motion of the torus isomer is generated by coherent superposition of many one-particle-one-hole excitations across the sloping Fermi surface that strongly violates the time-reversal symmetry. By comparing results of the TDHF and the RPA calculations, we find that the precession motion obtained by the TDHF calculation is a pure collective motion well decoupled from other collective modes.
\end{abstract}

PACS numbers: 21.60.Jz, 21.60.Ev, 27.40.+z

Nuclear rotation is a collective motion that restores the symmetry spontaneously broken in the self-consistent mean field. When deformed nuclei break the spherical symmetry but preserve the axial symmetry, the rotation about the symmetry $(z)$ axis is quantum mechanically forbidden. For instance, in high-spin oblate isomer states, the total angular momentum about the symmetry axis is constructed not by the collective rotation but the alignments of the angular momenta of individual nucleons [1, 2]. However, even such a state can rotate about an axis perpendicular to the symmetry axis, because the density distribution breaks the rotational symmetry about that axis. Below we call this $(x$ or $y)$ axis a perpendicular axis. This rotational degree of freedom causes the precession motion of the system as a whole [3].

In our previous paper [4], we showed the existence of a stable torus configuration in high-spin excited states of ${ }^{40} \mathrm{Ca}$, whose $z$ component of the total angular momentum, $K$, is $K=60 \hbar$. This large angular momentum is generated by alignment of single-particle angular momenta of totally twelve nucleons; the $z$ components of the orbital angular momenta, $\Lambda=+4,+5$, and $+6 \hbar$ for spin-up or -down neutrons and protons, are summed up to $K=60 \hbar$. Thus, this torus isomer has a significant amount of circulating current. A question then arises how such a "femto-scale magnet" rotates collectively to restore the broken symmetry about a perpendicular axis.

A key physical quantity to understand fundamental properties of nuclear rotation is the moment of inertia about a perpendicular axis. It is theoretically known that an independent-particle configuration in a deformed harmonic-oscillator potential rotates with rigid moment of inertia, provided that the self-consistency between the potential and the density distribution is fulfilled [5]. In reality, however, measured moments of inertia for deformed nuclei largely deviate from the rigid-body values. For instance, measured moments of inertia for precession motions of high- $K$ prolate isomers are significantly smaller than rigid-body values [6, 7]. This reduction has been seen at high spin where pairing correlations are negligible and is attributed to shell effects [6]. For high- $K$ oblate isomers, precession modes have not yet been observed. A possible reason is that their moments of inertia are much reduced from rigid-body values due to oblate shell structure at small deformation [8]. Then excitation energies of precession motion become higher. This would be a reason why the search for precession modes of high- $K$ oblate isomer is difficult and remains as an experimental challenge. The high$K$ torus isomer can be regarded as an extreme limit of the high- $K$ oblate isomer. Therefore, dynamical properties of the high- $K$ torus isomer revealed in its moment of inertia about a perpendicular axis will provide a fresh insight into dynamical properties of high- $K$ oblate isomers as well.

In this Letter, we present a periodic numerical solution of the precession motion of the high- $K$ torus isomer in ${ }^{40} \mathrm{Ca}$ described by the three-dimensional time-dependent Hartree-Fock (TDHF) equation. We trigger the precession motion by applying a certain amount of angular momentum in the direction of a perpendicular axis. We estimate the moment of inertia characterizing such an exotic mode of nuclear collective rotation. We find that the obtained moment of inertia is close to the rigid-body value. This result is surprising, because the high- $K$ torus isomer is created by aligned angular momenta of independent particle motion and possesses strong time-odd components in the self-consistent mean field. We shall also analyze the microscopic structure of the precession motion using the random-phase approximation (RPA) method and compare with the result of the TDHF calculation.

Since the TDHF method describes time-evolution of a 


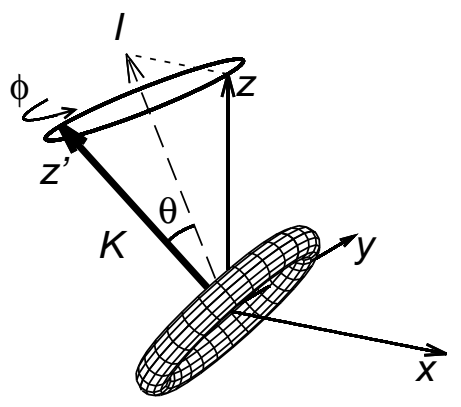

FIG. 1. Schematic picture of the precession motion of a high- $K$ torus isomer. The bold solid arrow denotes the symmetry axis of the density distribution. The dashed arrow denotes the precession axis. The symbols $\theta$ and $\phi$ denote the tilting and the rotation angles, respectively.

wave packet, quantization is necessary to obtain quantum spectra. If we succeed in obtaining periodic numerical solutions in real-time evolution of the TDHF mean field, then we can adopt the semi-classical quantization procedure. It is, however, very difficult to obtain the periodic solutions, because nonlinear effects tend to destroy the periodic motion and lead to chaotic motion. Thus, up to now, periodic solutions have been found only for a few relatively simple cases such as large-amplitude monopole vibrations in ${ }^{4} \mathrm{He}$ and ${ }^{16} \mathrm{O}$ [9]. Periodic solutions for rotational modes have not yet been reported. In this Letter, we will also show that the precession frequency obtained in the TDHF calculation agrees with that of the RPA method in good approximation. That is, we have, for the first time, succeeded in obtaining the quantum excitation energy of the precession motion by the numerical application of the TDHF method.

Figure 1 shows a schematic picture for the precession motion of a high- $K$ torus isomer. In the figure, the bold solid arrow denotes the symmetry $\left(z^{\prime}\right)$ axis of the density distribution in the body-fixed frame. At $t=0$, this axis is identical to the $z$ axis in the laboratory frame and the torus isomer has the angular momentum, $K$, along this axis. When we give an angular momentum to the (negative) direction of the $x$ axis (the dotted line) at $t=0$, the total angular momentum changes to $\vec{I}$. Then, the precession motion starts. The symmetry $\left(z^{\prime}\right)$ axis rotates about a fixed axis (the dashed arrow) that coincides with the direction of the total angular momentum $\vec{I}$. We call this axis 'the precession axis'. In the precession motion, the value $K$ is conserved. The tilting angle, $\theta$, is defined as the angle between the symmetry $\left(z^{\prime}\right)$ axis and the precession axis (the direction of the total angular momentum). The symbol $\phi$ denotes the rotation angle of the $z^{\prime}$ axis rotating about the precession axis. The moment of inertia for the rotation about a perpendicular axis of the torus configuration, $\mathscr{T}_{\perp}$, is then given by $\mathscr{T}_{\perp}=I / \omega_{\text {prec }}$, where $\omega_{\text {prec }}$ denotes the rotational frequency of the precession motion. The first excited state of the precession motion is the state with $I=K+1$. Since the torus isomer of ${ }^{40} \mathrm{Ca}$ has $K=60 \hbar$, we calculate the precession motion with $I=61 \hbar$.

To calculate the precession motion by means of the TDHF method, we use the code Sky3D [10]. We calculate the initial state of the torus configuration for ${ }^{40} \mathrm{Ca}$ with the $z$ component of the total angular momentum $K=60$ $\hbar$ by the cranked HF method using this code. The details are given in Ref. [4]. In the calculations, the single-particle wave functions are described on a Cartesian grid with a grid spacing of $1.0 \mathrm{fm}$. We take $32 \times 32 \times 24$ grid points for the $\mathrm{x}, \mathrm{y}$, and $\mathrm{z}$ directions, respectively. In all the calculations, we use the SLy6 interaction [11]. As shown in Ref. [4], the interaction dependence is negligible, because the spin-orbit force effects are weak in the torus configuration. The obtained density distribution on the plane at $z=0$ is well fitted by $\rho(r)=\rho_{0} e^{-\left(r-R_{0}\right)^{2} / \sigma^{2}}$, where $\rho_{0}=0.12$ $\mathrm{fm}^{-3}, R_{0}=6.06 \mathrm{fm}$, and $\sigma=1.64 \mathrm{fm}$. The rigid-body moment of inertia calculated using the obtained density distribution is $\mathscr{T}_{\perp}^{\text {rid }}=21.1 \hbar^{2} / \mathrm{MeV}$.

To excite the precession motion, we provide an impulsive force at $t=0 \mathrm{fm} / c$ by the external potential given by $V_{\text {ext }}(r, \varphi, z)=V_{0} z \cos \varphi \exp \left[-\left(r-R_{0}\right)^{2} / \sigma^{2}\right]$. The parameter $V_{0}$ is chosen such that the total angular momentum becomes $I=61 \hbar$, that is, the $x$ component of the total angular momentum, $I_{x}$, is $I_{x}=-11.0 \hbar$ at $t=0[I=$ $\left.\sqrt{60^{2}+(-11)^{2}} \hbar=61 \hbar\right]$. Here, we use $V_{0}=0.12757 \mathrm{MeV}$. We determine the time evolution of the density distribution by solving the TDHF equation of motion, $i \hbar \dot{\rho}=[h, \rho]$, where $h$ is the single-particle Hamiltonian and $\rho$ is the onebody density matrix. To solve the TDHF equation, we take Taylor expansion to the time-development operator up to the 12 order in the code. The time step of the TDHF calculations is $0.2 \mathrm{fm} / c$. We calculate the time-evolution until $3000 \mathrm{fm} / c$. Thus, we obtain about 7.5 periods of the precession motion.

Figure 2 shows snapshots of the time-evolution of the density distribution obtained by the TDHF calculations. In the figure, we plot the surface at the half of the maximum value of the density distribution. The time step of each snapshot is $50 \mathrm{fm} / \mathrm{c}$. We can clearly see in this figure about one period of the precession motion of the high- $K$ torus isomer of ${ }^{40} \mathrm{Ca}$.

Figure 3 shows the time-evolution of (a) the total angular momentum $I$, (b) the tilting angle $\theta$, and (c) the rotational angle $\phi$. In Fig. 3 (a), we see that the value of the total angular momentum converges to about $61 \hbar$, indicating that the TDHF calculations work well for long duration. The total energy also converges well. By the impulsive force, $-\partial V_{\text {ext }} / \partial z$, exerted at $t=0$, not only the precession motion but also other collective motions such as the $\gamma$ vibrations might be excited. However, the tilting angle fluctuates only slightly between $10^{\circ}$ and $11^{\circ}$ [see Fig. 3 (b)] indicating that the coupling effects between the precession motion and other collective modes are rather weak. In Fig. 3 (c), we see that the rotational angle linearly increases in each period. The obtained periods are 401.4, 

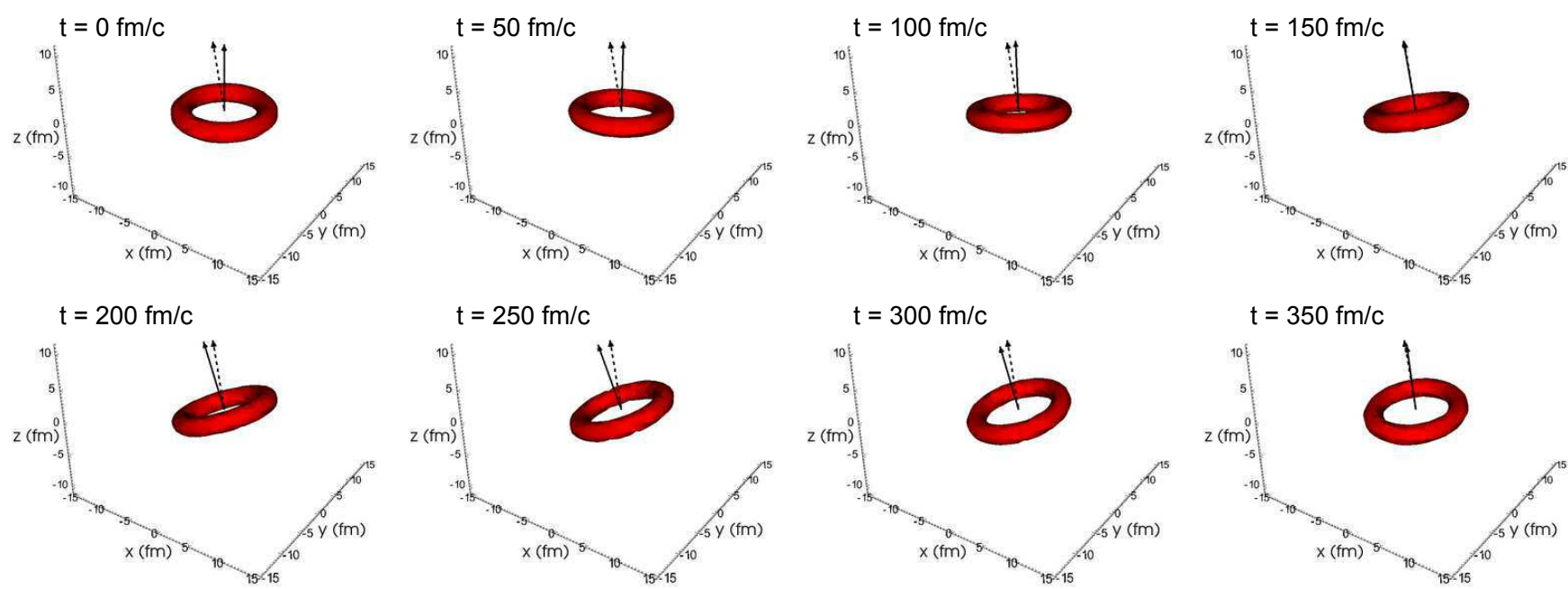

FIG. 2. (Color online) Snapshots of the time evolution of the density distribution of the high- $K$ torus isomer in ${ }^{40} \mathrm{Ca}$ obtained by the TDHF calculations. The (red) surface indicate that the density is half of the maximum value there. The time step of each snapshot is $50 \mathrm{fm} / c$. The solid and the dotted lines denote the symmetry and the precession axes, respectively.

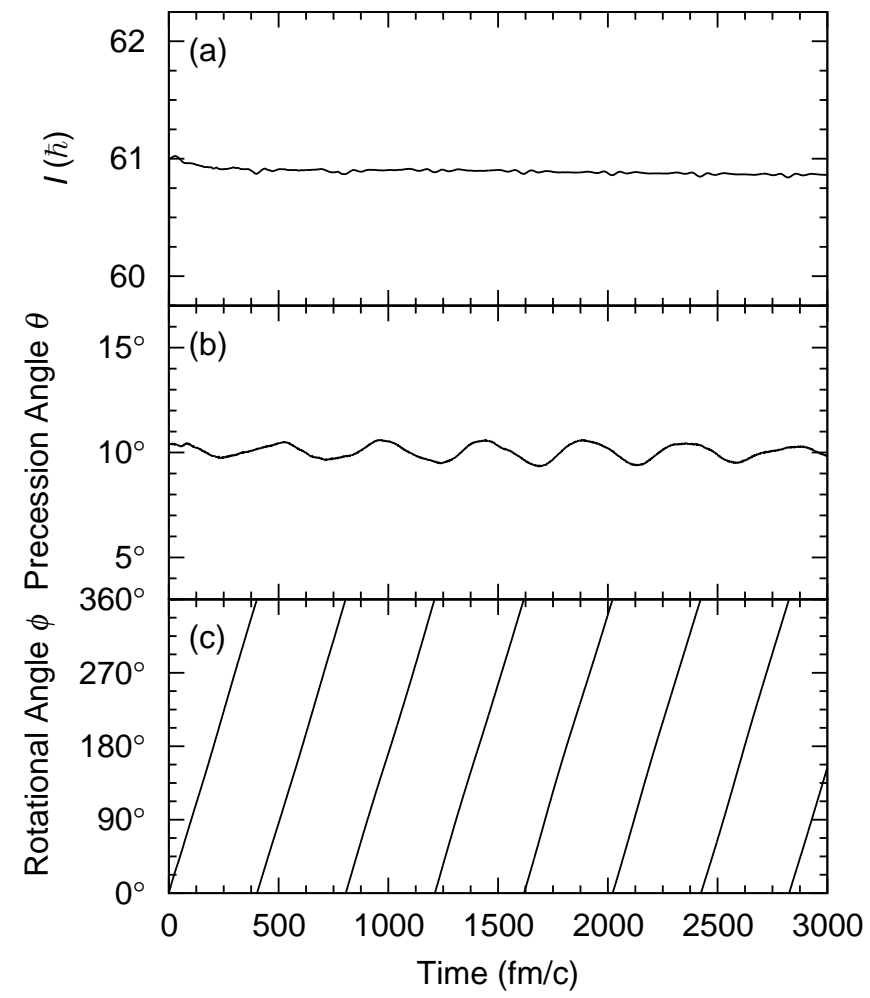

FIG. 3. Time-evolution of (a) total angular momentum $I$, (b) the tilting angle $\theta$, and (c) the rotational angle $\phi$ of the precession motion for the high- $K$ torus isomer in ${ }^{40} \mathrm{Ca}$.

403.5, 404.6, 405.4, 403.5, 400.9, and $401.5 \mathrm{fm} / c$. The fluctuations of the period indicate the extent of the effects due to the couplings with other collective modes and/or precision of numerical calculation. They are much smaller (less than $1 \%$ ) than the time scale of the precession mo- tion. The average period, $T_{\text {prec }}$, is $403.0 \mathrm{fm} / c$. Thus, the average frequency is $\omega_{\text {prec }}=2 \pi / T_{\text {prec }}=3.08 \mathrm{MeV} / \hbar$. We can identify $\hbar \omega_{\text {prec }}$ with the $\Delta I=1$ excitation energy of the precession mode of the high- $K$ torus isomer. We shall later confirm this interpretation in connection with the RPA treatment of this mode, The moment of inertia obtained in this way is $\mathscr{T}_{\perp}^{\mathrm{TDHF}}=I / \omega_{\text {prec }}=19.8 \hbar^{2} / \mathrm{MeV}$, which is very close to the rigid-body value $\mathscr{T}_{\perp}^{\text {rid }}=21.1 \hbar^{2} / \mathrm{MeV}$. The high- $K$ torus isomer is a unique quantum object characterized by the alignment of angular momenta of independent single-particle motions. The alignment causes a significant amount of circulating current and, as a consequence, the self-consistent mean field strongly violates the time-reversal symmetry. Although these features are essentially different from the classical rigid body, we find that the high- $K$ torus isomer performs a collective rotation about a perpendicular axis with the moment of inertia close to the rigid-body value.

To obtain a deeper understanding of the microscopic particle-hole structure generating the collective precession motion, we have performed an RPA calculation with the radial displaced harmonic-oscillator (RDHO) model [12]. We also confirm the validity of the relation $\mathscr{T}_{\perp}^{\mathrm{TDHF}}=$ $I / \omega_{\text {prec }}$ used to extract the moment of inertia from the realtime TDHF evolution. The RDHO model represents the major features of the torus isomer and works well, because effects of the spin-orbit force are negligible in the torus configuration. We can also avoid the complications for the treatment of unbound single-particle states by using this model. In the RDHO model for the torus configuration, the single-particle potential $V$ is given, in the cylindrical coordinates, by $V(r, z)=\frac{1}{2} m \omega_{0}^{2}\left(r-R_{0}\right)^{2}+\frac{1}{2} m \omega_{0}^{2} z^{2}$, where $m$ and $R_{0}$ denote the nucleon mass and the torus radius, respectively. The Coulomb potential is ignored for 
simplicity. The harmonic-oscillator frequency $\omega_{0}$ is determined such that the density distribution calculated with this model agrees, in good approximation, with that of the torus isomer obtained by the cranked HF calculation. The rigidbody moment of inertia calculated with the RDHO density distribution is $\mathscr{T}_{\perp}^{\mathrm{RDHO}}=21.1 \hbar / \mathrm{MeV}$, which agrees with the rigid-body value, $\mathscr{T}_{\perp}^{\text {rid }}$, for the torus isomer.

We can easily determine the frequency of the precession motion by solving the RPA dispersion equation [7], $\mathscr{T}_{\perp}^{\mathrm{RPA}}(\omega)=K / \omega$, where the moment of inertia, $\mathscr{T}_{\perp}^{\mathrm{RPA}}$, is a function of $\omega$ defined by

$$
\mathscr{T}_{\perp}^{\mathrm{RPA}}(\omega)=\frac{\hbar^{2}}{2} \sum_{p h}\left\{\frac{\left|J_{+}^{p h}\right|^{2}}{\left(\epsilon_{p h}-\hbar \omega\right)}+\frac{\left|J_{-}^{p h}\right|^{2}}{\left(\epsilon_{p h}+\hbar \omega\right)}\right\} .
$$

Here the sum is taken over the one-particle (1p)-one-hole (1h) excitations across the sloping Fermi surface (see Fig. 3 in Ref. [4]) and $\epsilon_{p h}$ denote their excitation energies. The quantities $J_{ \pm}^{p h}$ represent the matrix elements of the angular momentum raising and lowering operators, $J_{ \pm}^{p h}=$ $\left\langle p h\left|\hat{J}_{ \pm}\right| 0\right\rangle$, between the torus configuration $|0\rangle$ and the $1 \mathrm{p}-$ $1 \mathrm{~h}$ excited states $|p h\rangle$. This RPA dispersion equation is valid for velocity-independent residual interactions, and it yields the classical relation, $\mathscr{T}_{\perp}^{\text {rid }}=I / \omega$, except that anharmonic effects higher order in $1 / K$ are ignored and, accordingly, $I$ is approximated by $K$. By solving the RPA dispersion equation, we can simultaneously determine the frequency, $\omega$, and the moment of inertia, $\mathscr{T}_{\perp}$, of the precession motion. The lowest eigen-frequency that satisfies the above equation is just the RPA precession frequency of interest. We denote this solution $\omega_{\mathrm{RPA}}$. The precession motion is generated by coherent superposition of many $1 \mathrm{p}-1 \mathrm{~h}$ excitations across the sloping Fermi surface. The value of $\mathscr{T}_{\perp}^{\mathrm{RPA}}$ at $\omega_{\mathrm{RPA}}$ is the RPA moment of inertia for the precession motion. In the limit $\omega_{\mathrm{RPA}}=0, \mathscr{T}_{\perp}^{\mathrm{RPA}}$ reduces to the adiabatic cranking formula, $\mathscr{T}_{\perp}^{\text {crank }}$. Using the singleparticle wave functions obtained by the RDHO model, we determine $\omega_{\mathrm{RPA}}$ and $\mathscr{T}_{\perp}^{\mathrm{RPA}}$. In the calculations, we take all $1 \mathrm{p}-1 \mathrm{~h}$ excitations whose energies are below $\epsilon_{p h} \leq 30 \mathrm{MeV}$. We obtain $\omega_{\mathrm{RPA}}=3.02 \mathrm{MeV} / \hbar$ and $\mathscr{T}_{\perp}^{\mathrm{RPA}}=19.6 \hbar^{2} / \mathrm{MeV}$. This value of the RPA moment of inertia is different from the adiabatic cranking value $\mathscr{T}_{\perp}^{\text {crank }}=20.0 \hbar^{2} / \mathrm{MeV}$ only slightly, indicating that the effect of the finite frequency $\left(\omega_{\mathrm{RPA}} \neq 0\right)$ is rather small for the precession motion under consideration.

The RPA frequency $\omega_{\mathrm{RPA}}$ and the moment of inertia $\mathscr{T}_{\perp}^{\mathrm{RPA}}$ agree with the TDHF results for $\omega_{\text {prec }}$ and $\mathscr{T}_{\perp}^{\mathrm{TDHF}}$ in very good approximation. If $K$ is replaced with $I=K+1$ in the RPA dispersion equation, the agreement becomes even better $\left(\omega_{\mathrm{RPA}}=3.07 \mathrm{MeV} / \hbar\right)$. This almost perfect agreement clearly indicates that the periodic numerical solution obtained in the real-time TDHF evolution describes the collective rotation well decoupled from other collective modes. The agreement between the TDHF and RPA results furthermore suggests that the net effect of the velocitydependent interactions such as the spin-orbit interaction is small, despite the presence of a significant amount of circulating current which strongly violates the time-reversal symmetry in the self-consistent mean field. As we have seen in [4], the effect of the spin-orbit potential almost cancel out between the inside and outside of the torus radius $R_{0}$. This suggests that the velocity-dependent interaction effects are almost canceled out for the precession motion under consideration. The results of the TDHF and the RPA calculations thus suggest that basic physical conditions for the occurrence of the rigid precession motion are 1) the independent-particle configuration is pure and stable, 2) the symmetry breaking about a perpendicular axis is sufficiently strong, and 3) the net effect of the velocitydependent interactions is small.

In summary, we have obtained a periodic numerical solution in the TDHF time-evolution, that describes the precession motion of the high- $K$ torus isomer with $K=60$ $\hbar$ in ${ }^{40} \mathrm{Ca}$. Although the high- $K$ torus isomer is a unique quantum object characterized by the alignment of angular momenta of independent single-particle motions, we find that the torus isomer performs a collective rotation about an axis perpendicular to the symmetry axis with the moment of inertia close to the classical rigid-body value. We have also performed the RPA calculation for the precession motion with the RDHO model. By comparing the results of the TDHF and the RPA calculations, we have confirmed that the periodic TDHF solution corresponds to the precession mode generated by coherent superposition of many $1 \mathrm{p}-1 \mathrm{~h}$ excitations across the sloping Fermi surface. This exotic mode of rotation at high spin is ideally decoupled from other collective modes.

A part of this research has been funded by MEXT HPCI STRATEGIC PROGRAM. This work was undertaken as part by the Yukawa International Project for Quark-Hadron Sciences (YIPQS). J.A. M. was supported by BMBF under contract number 06FY9086 and 05P12RFFTG, respectively.

[1] A. Bohr, Proc. Int. Physics 'Enrico Fermi', Cours LXIX, ed. A. Bohr and R. A. Broglia (Amsterdam: North-Holland, 1977), p3.

[2] A.V. Afanasjev et al., Phys. Rep. 322, 1 (1999).

[3] A. Bohr and B.R. Mottelson, Nucl. Phys. A354, 303c (1981).

[4] T. Ichikawa et al., Phys. Rev. Lett. 109, 232503 (2012).

[5] A. Bohr and B.R. Mottelson, "Nuclear Structure" Vol. I.

[6] M.A. Deleplanque et al., Phys. Rev. C 69, 044309 (2004).

[7] Y.R. Shimizu et al., Phys. Rev. C 72, 014306 (2005).

[8] C.G. Anderson et al., Nucl. Phys. A 361, 147 ( 1981).

[9] J.-S. Wu et al., Phys. Rev. C 60, 044302 (1999).

[10] J.A. Maruhn et al., arXive:1310.5946.

[11] E. Chabanat et al., Nucl. Phys. A627, 710 (1997).

[12] C.Y. Wong, Ann. Phys. 77, 279 (1973). 